\title{
A comparison of obesity indices in relation to mortality in type 2 diabetes: the Fremantle Diabetes Study
}

\author{
Joel Tate ${ }^{1}$ - Matthew Knuiman ${ }^{1}$ - Wendy A. Davis ${ }^{2} \cdot$ Timothy M. E. Davis $^{2}$ - David G. Bruce ${ }^{2}$ \\ Received: 26 September 2019 / Accepted: 5 November 2019 / Published online: 14 December 2019 \\ (C) Springer-Verlag GmbH Germany, part of Springer Nature 2019
}

\begin{abstract}
Aims/hypothesis This prospective association study aimed to compare the relationship between each of four obesity indices and mortality risk in people with type 2 diabetes.

Methods The associations of BMI, waist circumference, WHR and A Body Shape Index (ABSI) with all-cause mortality were analysed in 1282 participants of the Fremantle Diabetes Study, followed for up to 20 years after baseline assessment. Models were adjusted for age and other confounders; assessments as continuous measures and by quintile were carried out for men and women separately. Sensitivity analyses were conducted to minimise reverse causality.

Results When indices were assessed as continuous variables, there were significant bivariate associations with mortality for: ABSI, which was greater in both men and women who died $(p<0.001)$; WHR, which was greater in women only $(p=0.033)$; and BMI, which was lower in women only $(p<0.001)$. When assessed by quintile, there were significant bivariate associations with mortality for ABSI in men and women $(p<0.001)$ and BMI in women only $(p=0.002)$. In Cox models of time to death, adjusted for age, diabetes duration, ethnicity and smoking, ABSI quintiles showed a linear trend for both men $(p=0.003)$ and women $(p=0.035)$. Men in the fifth ABSI quintile had an increased mortality risk compared with those in the first quintile (HR [95\% CI]: $1.74[1.24,2.44])$ and women in the fifth ABSI quintile had an increased mortality risk that approached statistical significance $(1.42$ [0.97, 2.08], $p=0.08)$. Men in the fifth WHR quintile had an increased mortality risk $(1.47$ [1.05, 2.06]). There was no association between mortality and BMI or waist circumference in either sex.

Conclusions/interpretation ABSI was the obesity index most strongly associated with all-cause mortality in Australians with type 2 diabetes. There was no evidence for an obesity paradox with any of the assessed indices. ABSI may be a better index of central obesity than waist circumference, BMI or WHR when assessing mortality risk in type 2 diabetes.
\end{abstract}

Keywords A Body Shape Index · Body Mass Index · Cohort study $\cdot$ Longitudinal study $\cdot$ Mortality $\cdot$ Obesity paradox $\cdot$ Type 2 diabetes $\cdot$ Waist Circumference $\cdot$ Waist-Hip ratio

\section{Abbreviations}

ABSI A Body Shape Index

ACR Albumin/creatinine ratio

ADL Activities of daily living

Electronic supplementary material The online version of this article (https://doi.org/10.1007/s00125-019-05057-8) contains peer-reviewed but unedited supplementary material, which is available to authorised users.

David G. Bruce

david.bruce@uwa.edu.au

1 School of Population and Global Health, University of Western Australia, Nedlands, WA, Australia

2 Medical School, Fremantle Hospital, PO Box 480, Fremantle, WA 6959, Australia

$\begin{array}{ll}\text { eGFR } & \text { Estimated GFR } \\ \text { FDS } & \text { Fremantle Diabetes Study } \\ \text { PAD } & \text { Peripheral arterial disease } \\ \text { WADLS } & \text { WA Data Linkage System }\end{array}$

\section{Introduction}

Obesity is associated with mortality in the general population. Individuals with a normal BMI have the highest survival rate, and mortality rates increase by $30 \%$ for each $5 \mathrm{~kg} / \mathrm{m}^{2}$ increment in BMI [1, 2]. Controversially, a number of studies have concluded that being overweight or mildly obese confers a survival advantage over having a normal BMI [3, 4]. This so-called obesity paradox was seen mainly in people with 


\section{Research in context}

\section{What is already known about this subject?}

- The relationship between obesity in type 2 diabetes and mortality is complex

- Some studies suggest that mild obesity may confer a survival advantage over normal body weight (the obesity paradox)

- The majority of studies have used BMI, but other obesity indices may better reflect differences in body composition, particularly central obesity

\section{What is the key question?}

- Which of the four obesity indices compared (BMI, waist circumference, WHR and A Body Shape Index (ABSI)) is the best predictor of mortality in type 2 diabetes?

\section{What are the new findings?}

- $\quad \mathrm{ABSI}$ had the strongest association with mortality

- There was no evidence of an obesity paradox with any obesity index

How might this impact on clinical practice in the foreseeable future?

- $\quad \mathrm{ABSI}$ is easily derived from simple anthropometric measures and may more accurately reflect the relationship between obesity and clinical outcomes in type 2 diabetes than other commonly used indices of obesity

chronic medical conditions [4]. In people with type 2 diabetes, the available research on the obesity paradox has provided conflicting evidence. Several studies reported benefit from being overweight or mildly obese [5-7], whereas others found no such survival benefit [8-10]. A study using pooled datasets found a non-linear U-shaped association between BMI and mortality in diabetes; optimal survival rate was at a BMI of $33 \mathrm{~kg} / \mathrm{m}^{2}$ [11]. An accurate characterisation of the relationship between body weight and mortality is extremely important given the central role of weight control in diabetes prevention and management.

Several explanations have been invoked to explain the obesity paradox. These include shortcomings of the methods used in existing studies, such as limited adjustment for confounders and failure to exclude underweight participants who may have lost weight because of undetected medical conditions [5, 11-13]. Smoking is a particularly important confounder to include because of the strength of the association between smoking and mortality in type 2 diabetes [12]. Another important consideration is the method used to assess obesity. Most studies have utilised BMI, an obesity index that does not take account of differences in regional fat deposition [14]. Alternative measures have been suggested that may be better able to account for the degree of central abdominal adiposity; these include waist circumference, waist-to-hip ratio (WHR) and A Body Shape Index (ABSI) [15-17].

The aim of the present study was to investigate the associations between each of these four obesity measures (BMI, waist circumference, WHR, ABSI) and all-cause mortality in participants with type 2 diabetes recruited to the first phase of the Fremantle Diabetes Study (FDS), an observational study with 20 years of follow-up.

\section{Methods}

Participant recruitment The FDS is an observational cohort study of residents with known diabetes in an urban community of 120,097 people in the Australian state of Western Australia. Recruitment occurred between 1993 and 1996, and participants were offered annual assessments for the next 5 years; endpoints, including death, were subsequently ascertained through the WA Data Linkage System (WADLS) [18]. Descriptions of recruitment, sample characteristics including classification of diabetes type, and details of non-recruited individuals have been published [19]. Eligible residents who declined participation were a mean 1.4 years older than participants, but their sex distribution, the proportion with type 2 diabetes and their use of blood-glucose-lowering therapies were similar. Of 2258 identified and eligible individuals, 1426 $(63 \%)$ were recruited and underwent the baseline assessment. Of these individuals, 1296 had type 2 diabetes. Participants with type 2 diabetes who had complete baseline anthropometric data and who were not underweight (BMI $<18.5 \mathrm{~kg} / \mathrm{m}^{2}$ ) comprise the present study sample.

The FDS protocol was approved by the Human Rights Committee at Fremantle Hospital. The WA Health 
Department Health Research Ethics Committee approved linkage of FDS participants and outcomes through the WADLS. All participants gave written informed consent.

\section{Clinical assessment}

The baseline clinical assessment comprised a comprehensive questionnaire, a physical examination and relevant clinical investigations, and biochemical testing of fasting blood and early morning urine samples [19]. Details of medical conditions and their management, as well as demographic, socioeconomic, lifestyle and other data, were recorded. Biochemical testing employed standard automated methods. Analyses were carried out in a single nationally accredited laboratory.

Baseline complications of diabetes were identified using standardised definitions [20]. Micro- and macroalbuminuria were defined from early morning urine albumin/creatinine ratio (ACR) and estimated (e)GFR was calculated from serum creatinine [21]. Retinopathy was defined as one microaneurysm in either eye or worse and/or evidence of previous laser treatment on direct/indirect ophthalmoscopy and/or more detailed ophthalmological data in individuals assessed for photocoagulation. Participants were classified as having CHD if there was a history of myocardial infarction, angina, coronary artery bypass grafting or angioplasty. They were classified as having cerebrovascular disease if there was a history of stroke and/or transient ischaemic attack. Peripheral arterial disease (PAD) was defined as an ankle-brachial index $\leq 0.90$ or the presence of a diabetesrelated lower-extremity amputation [20]. The Charlson Comorbidity Index was used to assess the impact of nondiabetes-related comorbidity, with diabetes and its complications excluded.

The Hospital Morbidity Data Collection contains information regarding all hospitalisations in WA since 1970, while the Registry for Births, Deaths and Marriages contains information on deaths [18]. The FDS was linked to these databases through the WADLS to provide relevant information up to the end of December 2012. This data linkage, providing up to 20 years of follow-up from study entry, was used to confirm self-reported diagnoses and dates of death.

\section{Anthropometry}

Height $(\mathrm{cm})$, weight $(\mathrm{kg})$, waist circumference $(\mathrm{cm}$; at midpoint between iliac crest and last palpable rib) and hip circumference (cm; at the widest portion of the buttocks) were measured by trained research nurses to assess BMI, waist circumference, WHR and ABSI. For consistency in comparisons, all measures were assessed both as continuous variables and by sex-specific quintiles. The ABSI was developed to incorporate abdominal obesity and take account of BMI, waist circumference and height; it is calculated as [15]:

$\mathrm{ABSI}=$ waist circumference $/\left(\mathrm{BMI}^{2 / 3} \times\right.$ height $\left.^{1 / 2}\right)$.

Statistical analysis Analysis was performed using SPSS version 23.0 (IBM, Armonk, NY, USA) and StataIC 13.1 (StataCorp, College Station, TX, USA). Two group comparisons were made using Fisher's exact test for percentages, Student's $t$ test for normally distributed variables and the Mann-Whitney $U$ test for non-normally distributed values. A $p$ value $<0.05$ was taken as statistically significant.

Given the known sex differences in mortality, all survival rate analyses were conducted separately for men and women. To facilitate comparison between the different anthropometric analyses, ABSI was expressed as ABSI $\times 10^{3}$ throughout.

Cox proportional hazards models for time to death were fitted to obtain age-adjusted HRs initially (model 1). Models were then adjusted for potential confounding variables (model 2) and finally for additional variables likely to be on the causal pathway between obesity and mortality (model 3 ). Relevant variables for the models were selected where $p<0.20$ in bivariate statistics. Variables were categorised as being on the causal pathway between obesity and mortality where there was a recognised association with obesity [22-26]. Potential confounding variables considered included: diabetes duration, alcohol intake, smoking status (never, ex-smoker, current smoker), education level, English fluency, ethnic background and marital status. Variables potentially on the causal pathway between obesity and mortality included: $\mathrm{HbA}_{1 \mathrm{c}}$, Charlson Comorbidity Index, diabetes treatment, antihypertensive treatment, aspirin therapy, lipid-lowering therapy, lipid levels (total cholesterol, HDLcholesterol, triacylglycerol), systolic and diastolic BP, retinopathy, $\log _{e}$ (urinary ACR), cerebrovascular disease, CHD, PAD, eGFR, exercise, mobility difficulties and difficulty with activities of daily living (ADL). The age-adjusted HR was obtained for each quintile of baseline anthropometric measure.

As a non-linear association between BMI and mortality was anticipated [11], tests for linearity and curvature in trend across quintiles were performed for each anthropometric measure by fitting (adjusted) Cox models. These used the median of each anthropometric measure per quintile for the linear trend and the square of the median of each anthropometric measure per quintile for curvature.

Sensitivity analyses were performed in restricted groups: (1) Anglo-Celt and Southern Europeans alone to avoid measurement differences due to ethnic background; (2) participants who survived for 24 months from study entry; and (3) participants without a cancer history, to reduce the impact of any pre-existing wasting illness. Quintiles derived from the whole cohort were used in the sensitivity analyses for the purpose of comparison. 


\section{Results}

Participant characteristics Of 1296 study participants, 14 were excluded: seven with missing anthropometric data and seven who were underweight. This left a final sample of 1282 participants $(98.9 \%)$. Baseline clinical characteristics are summarised in Table 1 and baseline obesity measures are summarised in electronic supplementary material (ESM) Table 1.

Overall, 385 men (61.6\%) died during a mean follow-up of $12.3 \pm 6.2$ years and 341 women $(51.9 \%)$ died during a mean follow-up of $13.7 \pm 5.9$ years. Bivariate associations between the four baseline obesity measures and survival rate are presented in ESM Table 1. In both men and women, ABSI was significantly greater in those who died (both as continuous measures and as quintiles, all $p<0.001$ ). In women only, there were significant bivariate associations with BMI, greater in those who died (continuous measure, $p<0.001$; quintiles, $p=0.002$ ) and similarly with WHR (continuous measure only, $p=0.033$ ). Bivariate associations between non-obesity variables and mortality are presented in ESM Table 2. For men, the variables associated with survival rate were age, diabetes duration, $\mathrm{HbA}_{1 \mathrm{c}}$, education, ethnicity, Charlson Comorbidity Index, mobility, ADL status, diabetes medications, antihypertensive therapies, lipid-lowering agents, systolic BP, smoking status, nephropathy, PAD and cerebrovascular disease. For women, the list of associations was similar except for several additional variables (alcohol consumption, marital status, exercise, aspirin use and CHD) and some that were not associated (education, ethnicity, lipid-lowering agents and smoking status).

Baseline obesity and survival In Cox proportional hazards models, age and age squared significantly predicted mortality risk in both sexes $(p<0.001)$, and consequently all models were adjusted for both variables. The age-adjusted HRs for confounders and factors on the causal pathway between obesity and mortality risk were then assessed and all variables with an HR with $p<0.2$ were entered into relevant models. The confounders that were identified in this way were diabetes duration, ethnicity and smoking status. The potential causal factors that were identified were $\mathrm{HbA}_{1 \mathrm{c}}$, diabetes treatment, antihypertensive treatment, lipid-lowering treatment, systolic $\mathrm{BP}$, retinopathy, $\log _{e}$ (urinary ACR), cerebrovascular disease, CHD, PAD, eGFR, Charlson Comorbidity Index, exercise, mobility and ADL status.

Table 2 shows HRs with 95\% CIs for baseline anthropometric measures as quintiles and trend tests for linearity and curvature after adjustment for age alone (model 1), after additional adjustment for confounders (model 2 ) and after adjustment for age, confounders and causal factors (model 3).

In models assessing the impact of BMI, there were no significant associations for men, though the test of curvature
Table 1 Baseline clinical characteristics of the cohort by sex

\begin{tabular}{|c|c|c|}
\hline Characteristic & $\begin{array}{l}\text { Men } \\
(n=625)\end{array}$ & $\begin{array}{l}\text { Women } \\
(n=657)\end{array}$ \\
\hline Age, years & $63.8 \pm 10.7$ & $64.0 \pm 11.7$ \\
\hline Diabetes duration, years & $4.0[1.0-9.0]$ & $4.0[1.0-9.0]$ \\
\hline $\mathrm{HbA}_{1 \mathrm{c}}, \%$ & $7.4[6.4-8.8]$ & $7.4[6.4-8.9]$ \\
\hline $\mathrm{HbA}_{1 \mathrm{c}}, \mathrm{mmol} / \mathrm{mol}$ & $57[46-73]$ & $57[46-74]$ \\
\hline Alcohol, standard drinks/day & $0.3[0.0-1.5]$ & $0.0[0.0-0.1]$ \\
\hline Education $>$ primary, $\%$ & 78.1 & 74.1 \\
\hline English fluency, \% & 86.2 & 83.2 \\
\hline Charlson Comorbidity Index $0, \%$ & 69.1 & 74.1 \\
\hline Charlson Comorbidity Index 1-2, \% & 23.8 & 20.4 \\
\hline Charlson Comorbidity Index $\geq 3, \%$ & 7.0 & 5.5 \\
\hline Anglo-Celtic ethnicity, $\%$ & 61.4 & 61.0 \\
\hline Southern European ethnicity, $\%$ & 17.0 & 18.9 \\
\hline Other European, \% & 9.4 & 7.6 \\
\hline Asian, \% & 3.4 & 3.2 \\
\hline Mixed/other, \% & 7.7 & 7.5 \\
\hline Indigenous, $\%$ & 1.1 & 1.8 \\
\hline Married, \% & 73.9 & 58.0 \\
\hline Recent exercise, $\%$ & 76.0 & 68.9 \\
\hline Mobility difficulties, $\%$ & 20.5 & 21.5 \\
\hline ADL difficulties, $\%$ & 9.3 & 7.6 \\
\hline Diet-controlled diabetes, $\%$ & 32.5 & 31.3 \\
\hline Glucose-lowering agents, $\%$ & 56.2 & 56.3 \\
\hline Insulin \pm glucose-lowering therapy, $\%$ & 11.3 & 12.4 \\
\hline Antihypertensives, $\%$ & 45.4 & 56.5 \\
\hline Lipid-lowering therapy, $\%$ & 10.0 & 11.3 \\
\hline Aspirin therapy, $\%$ & 25.0 & 19.1 \\
\hline Systolic BP, mmHg & $151 \pm 23$ & $151 \pm 24$ \\
\hline Diastolic BP, mmHg & $82 \pm 11$ & $79 \pm 11$ \\
\hline Total cholesterol, mmol/1 & $5.2 \pm 1.0$ & $5.7 \pm 1.1$ \\
\hline HDL-cholesterol, mmol/1 & $0.97 \pm 0.29$ & $1.1 \pm 0.33$ \\
\hline Serum triacylglycerol, mmol/1 & $1.9(1.0-3.4)$ & $1.9(0.4-9.1)$ \\
\hline Smoking: never, $\%$ & 24.3 & 64.3 \\
\hline Smoking: ex, \% & 57.2 & 24.0 \\
\hline Smoking: current, $\%$ & 18.5 & 11.7 \\
\hline Retinopathy, \% & 31.2 & 23.7 \\
\hline Urinary ACR, mg/mmol & $3.0(0.6-14.4)$ & $3.1(1.9-5.2)$ \\
\hline eGFR, $\mathrm{ml} \mathrm{min} \min ^{-1} 1.73 \mathrm{~m}^{-2}$ & $74.7 \pm 19.3$ & $73.1 \pm 21.0$ \\
\hline Cerebrovascular disease, $\%$ & 12.2 & 14.9 \\
\hline Ischaemic heart disease, $\%$ & 43.4 & 34.3 \\
\hline PAD, $\%$ & 23.8 & 24.9 \\
\hline
\end{tabular}

Data are expressed as mean $\pm \mathrm{SD}$, geometric mean (SD range), median [interquartile range] or proportions, as appropriate

approached statistical significance after age adjustment. In women, the test of curvature was significant (model 2) or approached statistical significance (models 1 and 3 ). The fifth BMI quintile had the highest HR in each model, but this was only significant after full adjustment (model 3). In models 
Table 2 Cox proportional hazard models for all-cause mortality for BMI, waist circumference, WHR and ABSI by sex

\begin{tabular}{|c|c|c|c|c|c|c|}
\hline Index & Model 1 & $\mathrm{p}$ value & Model 2 & $\mathrm{p}$ value & Model 3 & $\mathrm{p}$ value \\
\hline \multicolumn{7}{|l|}{ BMI } \\
\hline \multicolumn{7}{|l|}{ Men } \\
\hline 1st quintile & 1 & & 1 & & 1 & \\
\hline 2nd quintile & $0.84(0.62,1.14)$ & 0.26 & $0.86(0.63,1.17)$ & 0.33 & $0.90(0.64,1.26)$ & 0.53 \\
\hline 3rd quintile & $0.84(0.61,1.15)$ & 0.28 & $0.82(0.59,1.12)$ & 0.21 & $0.75(0.53,1.06)$ & 0.11 \\
\hline 4th quintile & $0.87(0.63,1.19)$ & 0.37 & $0.90(0.65,1.23)$ & 0.51 & $0.75(0.52,1.07)$ & 0.12 \\
\hline 5 th quintile & $1.09(0.79,1.51)$ & 0.59 & $0.99(0.71,1.39)$ & 0.97 & $0.88(0.62,1.27)$ & 0.51 \\
\hline Linear trend & & 0.60 & & 0.98 & & 0.33 \\
\hline Curvature & & 0.052 & & 0.15 & & 0.12 \\
\hline \multicolumn{7}{|l|}{ Women } \\
\hline 1st quintile & 1 & & 1 & & 1 & \\
\hline 2nd quintile & $0.99(0.72,1.35)$ & 0.94 & $0.90(0.65,1.25)$ & 0.53 & $1.12(0.76,1.66)$ & 0.56 \\
\hline 3rd quintile & $0.95(0.68,1.33)$ & 0.77 & $0.89(0.63,1.26)$ & 0.53 & $0.95(0.64,1.42)$ & 0.81 \\
\hline 4th quintile & $0.82(0.59,1.15)$ & 0.25 & $0.78(0.55,1.10)$ & 0.16 & $0.96(0.64,1.46)$ & 0.87 \\
\hline 5th quintile & $1.29(0.90,1.85)$ & 0.17 & $1.29(0.89,1.87)$ & 0.19 & $1.59(1.02,2.49)$ & $0.042^{*}$ \\
\hline Linear trend & & 0.53 & & 0.71 & & 0.13 \\
\hline Curvature & & 0.07 & & $0.020^{*}$ & & 0.08 \\
\hline \multicolumn{7}{|l|}{ Waist circumference } \\
\hline \multicolumn{7}{|l|}{ Men } \\
\hline 1st quintile & 1 & & 1 & & 1 & \\
\hline 2nd quintile & $1.16(0.85,1.59)$ & 0.34 & $1.21(0.88,1.65)$ & 0.24 & $1.03(0.73,1.47)$ & 0.86 \\
\hline 3rd quintile & $0.89(0.64,1.23)$ & 0.48 & $0.96(0.68,1.34)$ & 0.68 & $0.80(0.56,1.15)$ & 0.23 \\
\hline 4th quintile & $1.11(0.81,1.51)$ & 0.53 & $1.11(0.81,1.53)$ & 0.81 & $0.93(0.65,1.33)$ & 0.69 \\
\hline 5th quintile & $1.27(0.92,1.76)$ & 0.15 & $1.20(0.86,1.68)$ & 0.86 & $0.90(0.62,1.30)$ & 0.57 \\
\hline Linear trend & & 0.24 & & 0.42 & & 0.42 \\
\hline Curvature & & 0.36 & & 0.83 & & 0.64 \\
\hline \multicolumn{7}{|l|}{ Women } \\
\hline 1st quintile & 1 & & 1 & & 1 & \\
\hline 2nd quintile & $1.23(0.89,1.72)$ & 0.21 & $1.14(0.82,1.60)$ & 0.44 & $1.00(0.67,1.49)$ & $>0.99$ \\
\hline 3rd quintile & $1.09(0.78,1.53)$ & 0.60 & $1.04(0.74,1.46)$ & 0.84 & $0.96(0.65,1.40)$ & 0.82 \\
\hline 4th quintile & $1.47(1.05,2.06)$ & $0.024^{*}$ & $1.34(0.95,1.90)$ & 0.10 & $1.32(0.88,1.97)$ & 0.18 \\
\hline 5th quintile & $1.18(0.82,1.70)$ & 0.36 & $1.15(0.79,1.67)$ & 0.45 & $1.22(0.78,1.90)$ & 0.39 \\
\hline Linear trend & & 0.21 & & 0.30 & & 0.22 \\
\hline Curvature & & 0.28 & & 0.56 & & 0.81 \\
\hline \multicolumn{7}{|l|}{ WHR } \\
\hline \multicolumn{7}{|l|}{ Men } \\
\hline 1st quintile & 1 & & 1 & & 1 & \\
\hline 2nd quintile & $1.11(0.81,1.53)$ & 0.50 & $1.26(0.92,1.74)$ & 0.15 & $1.14(0.80,1.62)$ & 0.47 \\
\hline 3rd quintile & $1.16(0.84,1.60)$ & 0.36 & $1.31(0.95,1.81)$ & 0.11 & $1.00(0.71,1.43)$ & 0.98 \\
\hline 4th quintile & $0.98(0.71,1.34)$ & 0.89 & $1.02(0.74,1.40)$ & 0.92 & $0.86(0.61,1.23)$ & 0.41 \\
\hline 5 th quintile & $1.44(1.03,2.01)$ & $0.033^{*}$ & $1.47(1.05,2.06)$ & $0.023^{*}$ & $0.98(0.67,1.43)$ & 0.67 \\
\hline Linear trend & & 0.13 & & 0.13 & & 0.47 \\
\hline Curvature & & 0.37 & & 0.87 & & 0.98 \\
\hline \multicolumn{7}{|l|}{ Women } \\
\hline 1st quintile & 1 & & 1 & & 1 & \\
\hline 2nd quintile & $1.03(0.73,1.45)$ & 0.88 & $1.02(0.72,1.45)$ & 0.91 & $0.88(0.59,1.31)$ & 0.53 \\
\hline 3rd quintile & $0.88(0.61,1.25)$ & 0.47 & $0.88(0.61,1.27)$ & 0.50 & $0.68(0.44,1.04)$ & 0.08 \\
\hline 4th quintile & $0.99(0.70,1.40)$ & 0.96 & $0.93(0.65,1.33)$ & 0.70 & $0.75(0.49,1.15)$ & 0.19 \\
\hline 5th quintile & $1.25(0.89,1.74)$ & 0.20 & $1.20(0.85,1.70)$ & 0.29 & $1.02(0.68,1.53)$ & 0.93 \\
\hline
\end{tabular}


Table 2 (continued)

\begin{tabular}{|c|c|c|c|c|c|c|}
\hline Index & Model 1 & $\mathrm{p}$ value & Model 2 & $\mathrm{p}$ value & Model 3 & $\mathrm{p}$ value \\
\hline Linear trend & & 0.22 & & 0.38 & & 0.92 \\
\hline Curvature & & 0.16 & & 0.15 & & $0.026^{*}$ \\
\hline \multicolumn{7}{|l|}{ ABSI } \\
\hline \multicolumn{7}{|l|}{ Men } \\
\hline 1st quintile & 1 & & 1 & & 1 & \\
\hline 2nd quintile & $1.23(0.86,1.76)$ & 0.26 & $1.38(0.96,1.98)$ & 0.08 & $1.27(0.86,1.88)$ & 0.24 \\
\hline 3rd quintile & $1.12(0.79,1.61)$ & 0.52 & $1.29(0.90,1.85)$ & 0.17 & $1.13(0.76,1.67)$ & 0.55 \\
\hline 4th quintile & $1.20(0.85,1.70)$ & 0.31 & $1.35(0.95,1.92)$ & 0.10 & $1.12(0.76,1.66)$ & 0.56 \\
\hline 5 th quintile & $1.73(1.23,2.42)$ & $0.002^{*}$ & $1.74(1.24,2.44)$ & $0.001^{*}$ & $1.29(0.88,1.91)$ & 0.19 \\
\hline Linear trend & & $0.002^{*}$ & & $0.003^{*}$ & & 0.36 \\
\hline Curvature & & 0.24 & & 0.96 & & 0.99 \\
\hline \multicolumn{7}{|l|}{ Women } \\
\hline 1st quintile & 1 & & 1 & & 1 & \\
\hline 2nd quintile & $1.13(0.76,1.68)$ & 0.56 & $1.03(0.69,1.55)$ & 0.87 & $0.89(0.56,1.39)$ & 0.60 \\
\hline 3rd quintile & $1.30(0.89,1.91)$ & 0.18 & $1.12(0.75,1.66)$ & 0.58 & $1.04(0.67,1.59)$ & 0.87 \\
\hline 4th quintile & $1.35(0.93,1.97)$ & 0.12 & $1.20(0.81,1.76)$ & 0.36 & $1.11(0.72,1.71)$ & 0.63 \\
\hline 5th quintile & $1.62(1.12,2.34)$ & $0.011^{*}$ & $1.42(0.97,2.08)$ & 0.08 & $1.13(0.74,1.72)$ & 0.57 \\
\hline Linear trend & & $0.005^{*}$ & & $0.035^{*}$ & & 0.34 \\
\hline Curvature & & 0.99 & & 0.63 & & 0.81 \\
\hline
\end{tabular}

Data are shown for first to fifth quintiles

Model 1 is adjusted for age and age squared. Model 2 is additionally adjusted for confounders (diabetes duration, ethnicity, smoking). Model 3 is fully adjusted for age, confounders and possible causal factors on the pathway between obesity and mortality

* Statistically significant, $p<0.05$

assessing waist circumference, there were no consistent associations with mortality risk. In models assessing WHR, men in the fifth quintile had a significantly increased HR after adjustment for age (model 1: $1.44[1.03,2.01], p=0.033$ ) and confounders (model 2: 1.47 [1.05, 2.06], $p=0.023$ ), but there were no associations in women.

In models assessing ABSI, there were significant linear associations for both sexes in models after adjustment for age and confounders (models 1 and 2). In each model, the fifth quintile had the highest HR. The association with mortality risk was significant in men after adjustment for age (model 1: HR 1.73 [95\% CI 1.23, 2.42], $p=0.002$ ) and confounders (model 2: HR 1.74 [95\% CI 1.24, 2.44], $p=0.001$ ). The association was significant in women after age adjustment alone (model 1: HR 1.62 [95\% CI 1.12, 2.34], $p=0.011$ ) and approached statistical significance in model 2 (HR 1.42 [95\% CI $0.97,2.08], p=0.08$ ). The sensitivity analyses generated similar patterns of results for all anthropometric measures (see ESM Tables 3-6).

\section{Discussion}

In the present study, we investigated the associations between four commonly used obesity indices and long-term survival in type 2 diabetes. The study cohort was comprehensively assessed, allowing the analysis to take account of important confounding variables. There were substantial differences found among the indices examined. In men, WHR and ABSI were significantly associated with mortality after adjustment for confounders: those in the fifth quintile had an increased HR compared with those in the first quintile (mean HRs of 1.47 and 1.74, respectively). In women, those in the fifth quintile of ABSI had an increased HR (mean HR 1.42) with a trend towards statistical significance, but there was no association with WHR. No associations with mortality were seen for BMI or waist circumference, and there was no evidence of an obesity paradox with any index, though the association between BMI and mortality in women was non-linear.

ABSI had the strongest association with mortality in our cohort. While the association in women was attenuated after adjustment for confounders, the data suggest that a larger sample size could have achieved a statistically significant association. The associations with mortality that were found were considerably attenuated after adjustment for potential causal factors, providing a mechanistic explanation for them. ABSI has been shown to be associated with all-cause mortality in general population studies but has not previously been assessed in type 2 diabetes [27]. Most previous ABSI studies in samples from the general population reported similar 
findings to those of the present study, with mortality associations limited to those with the highest degrees of central obesity [28-34]. Associations with mortality were stronger for ABSI than for BMI [30, 34, 35], WHR [30, 32] or waist circumference $[31,35,36]$. Fewer studies have explored sex differences, but a Swedish study reported associations between ABSI and mortality in both men and women [32], supporting the possibility that the lack of an association for women in the present study reflected the limited study power. ABSI is derived from waist circumference adjusted for both height and weight; it was originally designed to assess the impact of central obesity while taking account of body size [15]. The findings of the present study suggest that ABSI may be a more accurate measure of body weight-associated mortality risk in type 2 diabetes than other commonly used indices of obesity.

The lack of evidence for an obesity paradox in the present study may be explained by the efforts taken to avoid reverse causation [37] by eliminating underweight participants at baseline and controlling for important confounders, including current/prior smoking, that could affect the association between obesity and mortality via collider bias $[12,38]$. The present data lend support to the contention that the obesity paradox may be explained by an intrinsic deficiency in BMI. BMI is a weak discriminator of body composition differences as it is central rather than peripheral fat that plays a dominant role in cardiometabolic risk [14].

Why the mortality associations with waist circumference and WHR were absent or weak in the present study is unknown, given previous findings [16]. Both waist circumference and WHR are sensitive to body size as well as the percentage and distribution of fat [15], and waist circumference and BMI are highly correlated [39]. ABSI is based on waist circumference and is approximately independent of height, weight and BMI [15]. In general population studies, ABSI consistently outperforms BMI, waist circumference and WHR in predicting all-cause mortality [27]. In one study, waist circumference was associated with mortality in a large population sample but not in those with diabetes [17]. The mortality associations seen with waist circumference, WHR and ABSI tended to be stronger in men than in women, consistent with a previous study [16], presumably reflecting sex differences in central body fat distribution.

The main study limitation relates to the small sample size compared with previous studies that have utilised large combined datasets or national population samples [3, 4]. Limited study power may underlie some of our findings, including the lack of association between mortality and either waist circumference or WHR. Cohort studies such as the FDS have the potential for recruitment and survivor biases. The timing of our study may also be a limitation, as when the FDS cohort was recruited there was less emphasis on cardiovascular risk factor management and the restricted diabetes treatment options included sulfonylureas and insulin, which promote weight gain.

The strengths of the present study include the representative nature of the cohort recruited from a postcode-defined region and the comprehensive clinical assessment that permitted adjustment for important confounders and potential causal factors. The anthropometric measures were obtained in a standardised fashion by trained research nurses. The study had an extended period of follow-up and the ability to access the WADLS permitted accurate assessment of confirmatory diagnoses and deaths.

In conclusion, central obesity was most strongly associated with excess mortality in type 2 diabetes when assessed by ABSI and the effect was seen in those in the fifth quintile. ABSI may be a better index of the proportionate relationship between adiposity and mortality in type 2 diabetes and should be considered for use in routine clinical care as a result.

Acknowledgements The authors wish to thank the staff at the Western Australian Data Linkage Branch, the Hospital Morbidity Data Collection and the Registry for Births, Deaths and Marriages.

Data availability Fremantle Diabetes Study data are available from the authors on request. Outcome data are available through additional separate application to the WA Data Linkage Service.

Funding The study was funded by the Raine Medical Research Foundation, University of Western Australia.

Duality of interest The authors declare that there is no duality of interest associated with the manuscript.

Contribution statement JT conceived the present study, conducted data analysis, drafted the manuscript and approved the final version. MK analysed data, assisted with the manuscript writing and approved the final version. WAD assisted with data acquisition, conducted the data linkage, assisted with data analysis and with the manuscript and approved the final version. TMED and DGB assisted with data interpretation and manuscript preparation and approved the final version. DGB is responsible for the integrity of the work as a whole.

\section{References}

1. Prospective Studies Collaboration, Whitlock G, Lewington S et al (2009) Body-mass index and cause-specific mortality in $900 \quad 000$ adults: collaborative analyses of 57 prospective studies. Lancet 373(9669):1083-1096. https://doi.org/10.1016/S0140-6736(09) 60318-4

2. de Gonzalez AB, Hartge P, Cerhan JR et al (2010) Body-mass index and mortality among 1.46 million white adults. N Engl J Med 363(23):2211-2219. https://doi.org/10.1056/NEJMoa1000367

3. Flegal KM, Kit BK, Orpana H, Graubard BI (2013) Association of all-cause mortality with overweight and obesity using standard body mass index categories: a systematic review and meta-analysis. JAMA 309(1):71-82. https://doi.org/10.1001/jama.2012.113905

4. The Global BMI Mortality Collaboration, Di Angelantonio E, Bhupathiraju SN et al (2016) Body-mass index and all-cause mortality: individual-participant-data meta-analysis of 239 
prospective studies in four continents. Lancet 388(10046):776786. https://doi.org/10.1016/S0140-6736(16)30175-1

5. Liu X-M, Zhan J, He Q-Q (2015) Overweight, obesity and risk of all-cause and cardiovascular mortality in patients with type 2 diabetes mellitus: a dose-response meta-analysis of prospective cohort studies. Eur J Epidemiol 30(1):35-45. https://doi.org/10.1007/ s10654-014-9973-5

6. Costanzo P, Cleland JGF, Pellicori P et al (2015) The obesity paradox in type 2 diabetes mellitus: relationship of body mass index to prognosis: a cohort study. Ann Intern Med 162(9):610-618. https:// doi.org/10.7326/M14-1551

7. Logue J, Walker JJ, Leese G et al (2013) Association between BMI measured within a year after diagnosis of type 2 diabetes and mortality. Diabetes Care 36(4):887-893. https://doi.org/10.2337/ dc12-0944

8. Hu G, Jousilahti P, Barengo NC, Qiao Q, Lakka TA, Tuomilehto J (2005) Physical activity, cardiovascular risk factors, and mortality among Finnish adults with diabetes. Diabetes Care 28(4):799-805. https://doi.org/10.2337/diacare.28.4.799

9. Katzmarzyk PT, Hu G, Cefalu WT, Mire E, Bouchard C (2013) The importance of waist circumference and BMI for mortality risk in diabetic adults. Diabetes Care 36(10):3128-3130. https://doi.org/ $10.2337 / \mathrm{dc} 13-0219$

10. McEwen LN, Karter AJ, Waitzfelder BE et al (2012) Predictors of mortality over 8 years in type 2 diabetic patients: Translating Research Into Action for Diabetes (TRIAD). Diabetes Care 35(6): 1301-1309. https://doi.org/10.2337/dc11-2281

11. Zaccardi F, Dhalwani N, Papamargaritis D et al (2017) Nonlinear association of BMI with all-cause and cardiovascular mortality in type 2 diabetes mellitus: a systematic review and meta-analysis of 414,587 participants in prospective studies. Diabetologia 60(2): 240-248. https://doi.org/10.1007/s00125-016-4162-6

12. Preston S, Stokes A (2014) Obesity paradox: conditioning on disease enhances biases in estimating the mortality risks of obesity. Epidemiology 25(3):454-461. https://doi.org/10.1097/EDE. 0000000000000075

13. Flegal KM, Graubard BI, Williamson DF, Cooper RS (2011) Reverse causation and illness-related weight loss in observational studies of body weight and mortality. Am J Epidemiol 173(1):1-9. https://doi.org/10.1093/aje/kwq341

14. Wells JC (2014) Commentary: The paradox of body mass index in obesity assessment: not a good index of adiposity, but not a bad index of cardio-metabolic risk. Int J Epidemiol 43(3):672-674. https://doi.org/10.1093/ije/dyu060

15. Krakauer NY, Krakauer JC (2012) A new body shape index predicts mortality hazard independently of body mass index. PLoS One 7(7):e39504. https://doi.org/10.1371/journal.pone. 0039504

16. Sluik D, Boeing H, Montonen J et al (2011) Associations between general and abdominal adiposity and mortality in individuals with diabetes mellitus. Am J Epidemiol 174(1):22-34. https://doi.org/10. 1093/aje/kwr048

17. Kahn HS, Bullard KM, Barker LE, Imperatore G (2012) Differences between adiposity indicators for predicting all-cause mortality in a representative sample of United States non-elderly adults. PLoS One 7(11):e50428. https://doi.org/10.1371/journal. pone. 0050428

18. Holman CD, Bass AJ, Rosman DL et al (2008) A decade of data linkage in Western Australia: strategic design, applications and benefits of the WA data linkage system. Aust Health Rev 32(4): 766-777. https://doi.org/10.1071/ah080766

19. Davis TME, Bruce DG, Davis WA (2013) Cohort profile: The Fremantle Diabetes Study. Int J Epidemiol 42(2):412-421. https:// doi.org/10.1093/ije/dys065
20. Norman PE, Davis WA, Bruce DG, Davis TM (2006) Peripheral arterial disease and risk of cardiac death in type 2 diabetes: the Fremantle Diabetes Study. Diabetes Care 29:575-580

21. Levey AS, Stevens LA, Schmid CH et al (2009) A new equation to estimate glomerular filtration rate. Ann Intern Med 150(9):604-612

22. Samper Ternent R, Al Snih S (2012) Obesity in older adults: epidemiology and implications for disability and disease. Rev Clin Gerontol 22(1):10-34. https://doi.org/10.1017/s0959259811000190

23. Fontana L, Hu F (2014) Optimal body weight for health and longevity: bridging basic, clinical, and population research. Aging Cell 13(3):391-400. https://doi.org/10.1111/acel.12207

24. Welborn TA, Dhaliwal SS, Bennett SA (2003) Waist-hip ratio is the dominant risk factor predicting cardiovascular death in Australia. Med J Aust 179(11-12):580-585

25. Khaw KT, Wareham N, Luben R et al (2001) Glycated haemoglobin, diabetes, and mortality in men in Norfolk cohort of European Prospective Investigation of Cancer and Nutrition (EPIC-Norfolk). BMJ 322(7277):15-18. https://doi.org/10.1136/ bmj.322.7277.15

26. Purnell JQ, Hokanson JE, Marcovina SM, Steffes MW, Cleary PA, Brunzell JD (1998) Effect of excessive weight gain with intensive therapy of type 1 diabetes on lipid levels and blood pressure: results from the DCCT. JAMA 280(2):140-146. https://doi.org/10.1001/ jama.280.2.140

27. Ji M, Zhang S, An R (2018) Effectiveness of A Body Shape Index (ABSI) in predicting chronic diseases and mortality: a systematic review and meta-analysis. Obesity Rev 19(5):737-759. https://doi. org/10.1111/obr.12666

28. Rahman SA, Adjeroh D (2015) Surface-Based Body Shape Index and its relationship with all-cause mortality. PLoS One 10(12): e0144639. https://doi.org/10.1371/journal.pone.0144639

29. Song X, Jousilahti P, Stehouwer CD et al (2015) Cardiovascular and all-cause mortality in relation to various anthropometric measures of obesity in Europeans. Nutr Metab Cardiovasc Dis 25(3):295-304. https://doi.org/10.1016/j.numecd.2014.09.004

30. Thomson CA, Garcia DO, Wertheim BC et al (2016) Body shape, adiposity index, and mortality in postmenopausal women: findings from the Women's Health Initiative. Obesity (Silver Spring) 24(5): 1061-1069. https://doi.org/10.1002/oby.21461

31. Krakauer NY, Krakauer JC (2014) Dynamic association of mortality hazard with body shape. PLoS One 9(2):e88793. https://doi.org/ 10.1371/journal.pone.0088793

32. Wierup I, Carlsson AC, Wandell P, Riserus U, Arnlov J, Borne Y (2015) Low anthropometric measures and mortality-results from the Malmo Diet and Cancer Study. Ann Med 47(4):325-331. https://doi.org/10.3109/07853890.2015.1042029

33. Sato Y, Fujimoto S, Konta T et al (2017) Body shape index: Sexspecific differences in predictive power for all-cause mortality in the Japanese population. PLoS One 12(5):e0177779. https://doi.org/10. 1371/journal.pone.0177779

34. Grant JF, Chittleborough CR, Shi Z, Taylor AW (2017) The association between A Body Shape Index and mortality: results from an Australian cohort. PLoS One 12(7):e0181244. https://doi.org/10. 1371/journal.pone. 0181244

35. Dhana K, Kavousi M, Ikram MA, Tiemeier HW, Hofman A, Franco OH (2016) Body shape index in comparison with other anthropometric measures in prediction of total and cause-specific mortality. J Epidemiol Community Health 70(1):90-96. https://doi. org/10.1136/jech-2014-205257

36. Davis WA, Chin E, Jee A et al (2010) Apolipoprotein E genotype and mortality in Southern European and Anglo-Celt patients with type 2 diabetes: the Fremantle Diabetes Study. Eur J Endocrinol 163(4):559-564. https://doi.org/10.1530/Eje-10-0474

37. Edqvist J, Rawshani A, Adiels M et al (2018) BMI and mortality in patients with new-onset type 2 diabetes: a comparison with age- and 
sex-matched control subjects from the general population. Diabetes Care 41(3):485-493. https://doi.org/10.2337/dc17-1309

38. Jenkins DA, Bowden J, Robinson HA et al (2018) Adipositymortality relationships in type 2 diabetes, coronary heart disease and cancer subgroups in the UK Biobank, and their modification by smoking. Diabetes Care. https://doi.org/10.2337/dc17-2508
39. Moore SC (2009) Waist versus weight: which matters more for mortality? Am J Clin Nutr 89(4):1003-1004. https://doi.org/10. 3945/ajcn.2009.27598

Publisher's note Springer Nature remains neutral with regard to jurisdictional claims in published maps and institutional affiliations. 